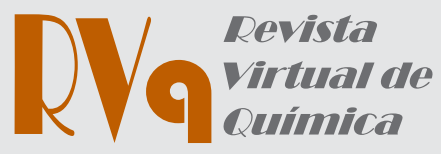

a Universidade Federal de Goiás, Departamento de Engenharia Florestal, CEP 74690-900, Goiânia-GO, Brasil.

*E-mail: raquel.menestrino@gmail.com

Recebido em: 2 de Abril de 2021

Aceito em: 20 de Abril de 2021

Publicado online: 28 de Julho de 2021

\section{Resíduo do Desdobro da Madeira de Hymenolobium petraeum Ducke para a Produção de Briquetes}

\author{
Briquette production with wood residues from Hymenolobium \\ petraeum Ducke
}

Raquel M. Ribeiro, ${ }^{a} *$ (1) Marco Antônio M. Bahia, ${ }^{a}$ Mateus Z. G. Caetano, ${ }^{a}$ Pedro Henrique C. V. Sousa, ${ }^{a}$ Rafaela Laís O. Fernandes, ${ }^{a}$ Mariana P. Franco, ${ }^{a}$ Carlos Roberto Sette Júnior ${ }^{a}$

\begin{abstract}
The processing operations of wood in sawmills generates a large amount of residues that can be used as raw material for briquettes production. The objective of this study was to evaluate the wood residues from Hymenolobium petraeum (Angelim Pedra) and its potential for briquettes production. The characterization was done with the granulometric profile, moisture content and bulk density of wood residue. Briquettes were produced and determined the apparent density and the durability. The wood residue from $\mathrm{H}$. petraeum presented bulk density of $204 \mathrm{~kg} \mathrm{~m}^{-3}$ and moisture content of $15.2 \%$ while briquettes produced showed bulk density of $1.336 \mathrm{~kg} \mathrm{~m}^{-3}$, moisture content of $11.8 \%$ and durability of $99.9 \%$. The residues generated during the processing of $H$. petroleum wood used in this study are suitable for production of high quality briquettes, being a viable technical alternative for its energy destination.
\end{abstract}

Keywords: Angelim Pedra; solid biofuel; energy resources; biomass

\section{Introdução}

O acelerado processo de urbanização mundial promove, entre outros, a pressão nos mais variados setores de produção, incluindo o energético. Diante desse cenário, a participação da biomassa na matriz energética brasileira e mundial vem crescendo nos últimos anos, com grande potencial para liderar o ranking de fontes renováveis, principalmente pela grande disponibilidade. ${ }^{1}$

Além de ser uma alternativa ao uso de fontes energéticas não renováveis, a biomassa vegetal vem sendo utilizada como insumo energético por diferentes segmentos industriais e domésticos ${ }^{2}$ devido a sua ampla abrangência, de organismos formados pela fotossíntese até os resíduos gerados a partir da sua utilização, como matéria orgânica oriunda de resíduos domésticos, comerciais e rurais e os resíduos florestais e agrícolas, incluindo os gerados no desdobro da madeira em serrarias. ${ }^{3}$

A biomassa florestal engloba todos os resíduos de plantios florestais, de serrarias de beneficiamento de madeira, indústrias de papel e celulose, fabricação de painéis, dentre outras. Nesses processos, a geração de resíduos representa um desperdício na produção, uma vez que nem sempre são convertidos em uma nova fonte de renda. ${ }^{4}$

Além disso, os resíduos, quando mal manejados e descartados em locais impróprios, podem se constituir em um passivo ambiental importante. Em 2010 foi instituída a Política Nacional de Resíduos Sólidos, pela Lei ${ }^{\circ} 12.305$, reunindo princípios, objetivos e diretrizes que visam a gestão integrada e o gerenciamento ambientalmente adequado dos resíduos sólidos. ${ }^{5}$ Assim, a partir de então, as empresas geradoras dos resíduos precisam dar um destino ambientalmente adequado, necessitando buscar alternativas de utilização para esse tipo de material.

Uma parte significativa da madeira desdobrada em serrarias no Brasil se transforma em resíduos, ${ }^{6}$ ou seja, não é aproveitada para sua finalidade principal. Essa grande quantidade de resíduo madeireiro pode ser aproveitada para a geração de energia na forma de biocombustíveis.?

$\mathrm{O}$ aproveitamento do resíduo madeireiro como fonte de energia se apresenta como uma alternativa viável para a redução dos impactos gerados pela sua produção, podendo ser utilizados como insumo tanto in natura como na forma de material densificado, na forma de pellets e briquetes. ${ }^{7} \mathrm{O}$ processo de densificação dos resíduos (produção dos briquetes) possui como principal vantagem a redução do seu volume, o alto valor e capacidade calorífica por volume, redução de custo com logística e transporte, estoque e manuseio facilitados e homogeneização do combustível. ${ }^{8,9}$ 
Este trabalho teve por objetivo avaliar as características do resíduo do desdobro da madeira Hymenolobium petraeum (Angelim Pedra) e o seu potencial para a produção de briquetes.

\section{Metodologia}

\subsection{Coleta do resíduo}

O resíduo utilizado neste estudo foi obtido do desdobro da madeira de $H$. petraeum em serraria. Toras sem casca da espécie foram desdobradas em serra fita e o resíduo deste processamento, constituído de serragem de madeira, foi coletado para a realização das análises descritas a seguir.

\subsection{Caracterização do resíduo}

Inicialmente, o resíduo madeireiro passou por uma classificação granulométrica, sendo submetido a uma separação mecânica utilizando peneiras classificadas em 20, 40, 60 e 100 mesh com auxílio de um agitador de peneiras com batidas intermitentes. Na sequência, foram determinados o teor de umidade e a densidade a granel, conforme preconizado pela ASTM E871- $2013^{10}$ e ABNT NBR $11941,{ }^{11}$ respectivamente.

\subsection{Produção dos briquetes}

A briquetagem do resíduo de madeira foi realizada em briquetadeira laboratorial, com temperatura de 120 ${ }^{\circ} \mathrm{C}$, pressão $140 \mathrm{kgf} \mathrm{cm}^{-2}$, tempo de compactação de 5 minutos e resfriamento de 5 minutos. As condições de briquetagem foram definidas experimentalmente a partir de testes preliminares de tempo de prensagem e de resfriamento. A pressão exercida está dentro da faixa utilizada na literatura ${ }^{13} \mathrm{e}$ a temperatura teve como objetivo a plasticização da lignina, ${ }^{12}$ atuando como ligante natural das partículas durante a compactação. Para cada briquete utilizou-se $40 \mathrm{~g}$ de resíduo, obtendo-se ao final um briquete de aproximadamente $4 \mathrm{~cm}$ de comprimento e $3 \mathrm{~cm}$ de diâmetro.
A densidade aparente foi determinada por meio do método estequiométrico, o qual consiste em obter o volume a partir de medições, utilizando um paquímetro e a massa do briquete em uma balança com precisão de 0,001 gramas. Foi obtida através da Equação 1:

$$
D A P=\frac{M i}{V}
$$

onde: DAP $=$ Densidade aparente $\left(\mathrm{g} \mathrm{cm}^{-3}\right) ; \mathrm{Mi}=$ Massa inicial $(\mathrm{g})$; V= Volume $\left(\mathrm{cm}^{3}\right)$.

A durabilidade foi determinada por perda de massa dos briquetes. ${ }^{14,15}$ Os briquetes foram pesados para a obtenção da massa inicial e na sequência colocados em uma peneira com tela de 2,83 mm de tamanho. Posteriormente, foram levados a um agitador de peneiras, permanecendo por 10 minutos, a 80 rotações por minuto. Após este procedimento, os briquetes foram novamente pesados e obtidos a massa final. A durabilidade foi calculada a partir da Equação 2:

$$
\text { Dur }=\frac{\text { Mid }- \text { Mfd }}{\text { Mid }} \times 100
$$

onde: Dur: Durabilidade do briquete (\%); Mid: Massa inicial (g); Mfd: Massa final (g).

\section{Resultados e Discussão}

3.1. Classificação granulométrica, densidade a granel e teor de umidade do resíduo

A caracterização do resíduo proveniente do desdobro da madeira de $H$. petraeum teve início pela sua classificação granulométrica, sendo observadas partículas de diferentes tamanhos (Figura 1). A maior parte das partículas foi classificada em 20 mesh (56\%), ou seja, considerado como "fino", ${ }^{16,13}$ uma vez que se encontra abaixo de $1 \mathrm{~mm}$.

Essa característica do resíduo é interessante para a produção de briquetes, uma vez que quanto menor o tamanho das partículas que compõem o resíduo, melhor será a sua compactação, com efeitos significativos e positivos

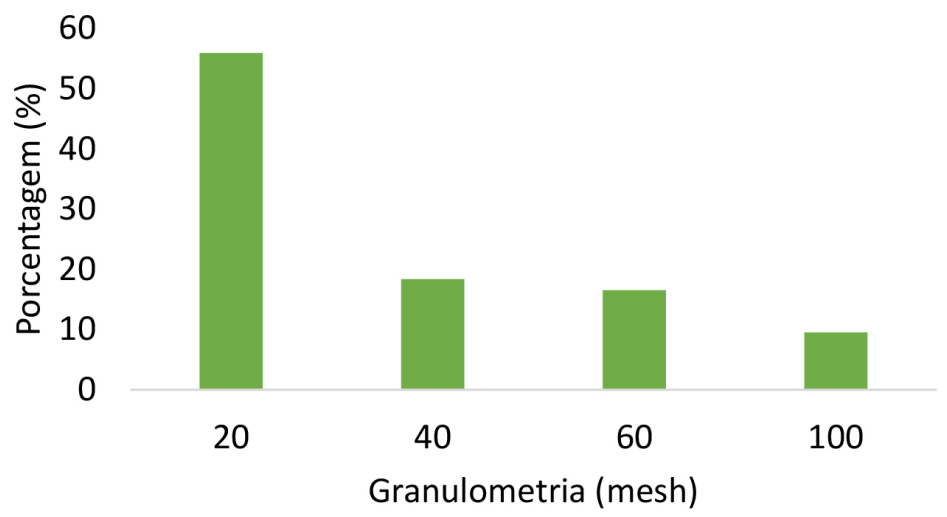

Figura 1. Perfil granulométrico do resíduo da madeira de H. petraeum 
na qualidade do biocombustível sólido. Desta forma, os resíduos gerados no desdobro da madeira utilizados neste estudo podem ser utilizados diretamente para a produção de briquetes, sem a necessidade de processamento, como trituração e moagem, reduzindo os custos associados ao seu aproveitamento energético.

Analisar o perfil granulométrico é de extrema importância pois a granulometria do resíduo interfere na resistência do briquete já que a presença de partículas menores ajuda a preencher espaços vazios. ${ }^{17,18}$

A densidade a granel do resíduo foi de $204 \mathrm{~kg} \mathrm{~m}^{-3}$. A determinação da densidade a granel é de fundamental importância para gerar informações úteis para a logística e transporte do resíduo. Os valores médios obtidos neste estudo indicam baixa densidade quando considerado o resíduo obtido diretamente do desdobro da madeira e assim, a sua compactação na forma de briquetes pode ser uma alternativa viável para o aumento da densidade e redução dos custos de transporte.

O teor de umidade é um importante parâmetro de um biocombustível sólido, uma vez que quanto menor a quantidade de água, melhor serão as suas características energéticas, especialmente o poder calorífico útil. ${ }^{19}$ Neste estudo, o resíduo apresentou teor de umidade de 15,2\%, estando dentro da faixa ideal para a produção de briquetes (8-15\%), ${ }^{20}$ não sendo necessária a aplicação de métodos de secagem antes da sua densificação, reduzindo os custos de produção do briquete.

\subsection{Densidade aparente, teor de umidade e durabilidade do briquete}

Foi possível produzir briquetes de qualidade com os resíduos do desdobro da madeira de $H$. petraeum. A densidade aparente média foi de $1.336 \mathrm{~kg} \mathrm{~m}^{-3}$, cerca de 7 vezes maior do que a densidade a granel do resíduo (204 $\mathrm{kg} \mathrm{m}^{-3}$ ), antes da compactação. Resultados semelhantes foram encontrados na literatura em briquetes produzidos a partir de outros resíduos madeireiros..$^{21,22}$

A densificação do resíduo madeireiro através da produção dos briquetes, promove um aumento na densidade; este incremento após a compactação, evidencia a importância dos processos de compactação dos resíduos para o melhor aproveitamento de materiais lignocelulósicos para a produção de bioenergia.

O teor de umidade dos briquetes, medido logo após a compactação do resíduo, indicou média de 11,8\%, ou seja, com uma redução de $3,4 \%$ em relação ao teor de umidade inicial $(15,2 \%)$. Esta pequena redução pode estar associada ao processo de produção do briquete, especialmente pela aplicação da temperatura de $120{ }^{\circ} \mathrm{C}$. De qualquer forma, conforme já discutido, a redução do teor de umidade do resíduo após a densificação é uma vantagem para a aplicação energética do biocombustível.

Os briquetes produzidos neste estudo apresentaram durabilidade média de $99,9 \%$, ou seja, caracterizados como pouco friáveis. ${ }^{23} \mathrm{~A}$ alta durabilidade dos biocombustíveis sólidos é importante para reduzir perdas de massa e de resistência mecânica ao longo do transporte e manuseio.

\section{Conclusão}

Os resíduos do desdobro da madeira de $H$. petraeum utilizados neste estudo são adequados para a produção de briquetes.

Os briquetes produzidos apresentaram alta qualidade, com densidade aparente de $1.336 \mathrm{~kg} \mathrm{~m}^{-3}$ e durabilidade de $99,9 \%$, sendo uma alternativa técnica viável para a destinação energética dos resíduos do desdobro da madeira de H. petraeum.

\section{Referências Bibliográficas}

1. Sítio da Indústria Brasileira de Árvores (IBÁ). Relatório 2020/ Ano base 2019. Disponível em: <https://iba.org/datafiles/ publicacoes/relatorios/relatorio-iba-2020.pdf>. Acesso em: 24 junho 2021.

2. Quirino, W. F.; Pinha, I. V. O.; Moreira, A. C. O. M.; Souza, F.; Tomazello Filho, M.; Densitometria de raios $\mathrm{X}$ na análise da qualidade de briquetes de resíduos de madeira. Scientia Forestalis 2012, 40, 96.[Link]

3. Brand, M. A.; Energia de biomassa florestal, 1a. ed., Interciência: Rio de Janeiro, 2010.

4. Sette Jr, C. R.; Moraes, M. D. A.; Coneglian, A.; Ribeiro, R. M.; Hansted, A. L. S.; Yamaji, F. M.; Forest harvest byproducts: use of waste as energy. Waste Management 2020, 114, 196. [CrossRef]

5. Sítio Presidência da República Casa Civil, Lei no 12.305 de 2 de agosto de 2010. Disponível em: <http://www.planalto.gov. br/ccivil 03/ ato2007-2010/2010/lei/112305.htm > Acesso em: 24 junho 2021.

6. Sítio Forestry Production and trade, Food and Agriculture Organization of the United Nations, 2016. Disponível em: $<$ https://www.oecd.org/gov/regulatory-policy/FAO_Full-Report. pdf $>$. Acesso em 24 junho 2021.

7. Dias, J. M. C. D. S.; Souza, D. T.; Braga, M.; Onoyama, M. M.; Miranda, C. H. B.; Barbosa, P. F. D.; Rocha, J. D.; Produção de briquetes e peletes a partir de resíduos agrícolas, adroindustriais e florestais. Disponível em: <https://www.infoteca.cnptia. embrapa.br/bitstream/doc/952626/1/DOC13.pdf>. Acesso em: 24 junho 2021.

8. Fernández, R. G.; Garcia, C. P.; Lavín, A. G.; Heras, J. L. B.; Pis, J. J.; Influence of physical properties of solid biomass fuels on the design and cost of storage installations. Waste Management 2013, 33, 1151. [CrossRef]

9. García-Maraver, A.; Rodriguez, M. L; Serrano-Bernardo, F; Diaz, L. F; Zamorano, M.; Factors affecting the quality of pellets made from residual biomass of olive trees. Fuel Processing Technology 2015, 129, 1. [CrossRef] 
10. ASTM E871-82, Standard Test Method for Moisture Content Analysis of Particulate Wood Fuels, ASTM International, West Conshohocken, PA, 2013. [Link]

11. ABNT, Associação Brasileira de Normas Técnicas NBR 11941/02. Determinação da densidade básica em madeira. Rio de Janeiro, RJ, Brasil: Associação brasileira de normas técnicas, 2013. [Link]

12. Chen, L.; Xing, L.; Han, L.; Renewable energy from agro -residues in China: solid biofuels and biomass briquetting technology. Renewable and Sustainable Energy Reviews 2009, 13, 2689. [CrossRef]

13. Quirino, W. F.; Pinha, I. V. O.; Moreira, A. C. O.; Souza, F.; Tomazello Filho, M.; Densitometria de raios X na análise da qualidade de briquetes de resíduos de madeira. Scientia Forestalis 2012, 40, 525. [Link]

14. Toscano, G.; Riva, G.; Foppa Pedretti, E.; Corinaldesi, F.; Mengarelli, C.; Duca, D.; Investigation on wood pellet quality and relationship between ash content and the most important chemical elements. Biomass and Bioenergy 2013, 56, 317. [CrossRef]

15. Liu, Z. J; Fei, B.; Jiang, Z.; Cai, Z.; Liu, X.; Important properties of bamboo pellets to be used as commercial solid fuel in China. Wood Science and Technology 2014, 48, 903. [CrossRef]

16. Bergström, D.; Israelsson, S.; Ohman, M.; Dahlqvist, S.-A.; Gref, R.; Boman, C.; Wasterlund, I. Effects of raw material particle size distribution on the characteristics of Scots pine sawdust fuel pellets. Fuel Processing Technology 2008, 89, 1324. [CrossRef]
17. Silva, D. A.; Yamaji, F. M.; Barros, J. L.; Roz, A. L.; Nakashima, G. T.; Caracterização de biomassas para briquetagem. Revista Floresta 2015, 45, 713. [CrossRef]

18. Nakashima, G. T; Dissertação de Mestrado, Universidade Federal de São Carlos, 2016. [Link]

19. Souza, M. M.; Da Silva, D. A.; Rochadelli, R.; Dos Santos, R.C.; Estimativa do poder calorífico e caracterização para uso energético de resíduos da colheita e do processamento de Pinus Taeda. Revista Floresta 2012, 42, 325. [CrossRef]

20. Silva, D. A.; Nakashima, G. T.; Barros, J. L.; Da Roz, A. L.; Yamaji, F. M.; Caracterização de biomassas para a briquetagem. Revista Floresta 2015, 45, 713. [CrossRef]

21. Dos Santos, R. C; Carneiro, A. C. O.; Damasceno, G. R. F; Castro, A. F. N. M; Castro, R. V. O; Da Costa, L. S.; De Lima Costa, S. E.; Efeito da variabilidade de resíduos madeireiros na produção e qualidade de briquetes. Advances in Forestry Science 2019, 6, 529. [CrossRef]

22. Paula, L. E. R; Trugilho, P. F; Rezende, R. N; Assis, C. O; Baliza, A. E. R.; Produção e avaliação de briquetes de resíduos lignocelulósicos. Pesquisa Florestal Brasileira 2011, 31, 103. [Link]

23. Sítio da Biblioteca Digital do Estado de Minas Gerais - Estudos preliminares de normalização de testes de controle de qualidade do carvão vegetal. Disponível em: $<$ http://www.bibliotecadigital. mg.gov.br/consulta/consultaDetalheDocumento. php?iCodDocumento=73944 > . Acesso em: 25 junho 2021 . 